\title{
Induction of oral tolerance to the acetylcholine receptor for treatment of myasthenia gravis
}

\author{
Howard L. Weiner \\ Brigham and Women's Hospital, Center for Neurologic Diseases, 77 Avenue Louis Pasteur, HIM 730, Boston, \\ Massachusetts 02115-5817, USA. Phone: (617) 525-5300; Fax: (617) 525-5252; E-mail: weiner@cnd.bwh.harvard.edu.
}

Autoimmune diseases involve a breakdown of the normal mechanisms of immune self tolerance. In recent years, it has become clear that the mechanisms by which peripheral immune tolerance is mediated are multiple, and include deletion, anergy, and the induction of active cellular regulation. Autoimmune diseases can broadly be classified into cell-mediated and humorally mediated diseases. Most are caused by Th1-type immune responses.

One of the best-characterized human autoimmune diseases is myasthenia gravis, in which there are both humoral and cellular immune responses against the acetylcholine receptor (AChR) (1). Anti-AChR antibodies are pathogenic: clinical diseases can be transferred from humans to animals by the passive transfer of antibodies from myasthenic patients to mice (2). Unequivocal proof of the autoimmune nature of putative human T-cell mediated autoimmune diseases such as multiple sclerosis is more difficult to demonstrate; it is obviously problematic to induce disease in animals by the adoptive transfer of human cells.

To date, treatment of autoimmune diseases in humans has relied primarily on general immunosuppression. One of the major goals of current immunologic investigation is to find approaches that specifically modulate or correct the abnormal pathogenic immune response without affecting the rest of the immune system. Oral administration of autoantigens to induce oral tolerance is 1 such approach.

Oral tolerance is a long-recognized method of inducing antigen-specific immune tolerance; it presumably evolved to prevent harmful reactions to ingested proteins (3). Although oral tolerance was originally described through experiments involving the feeding of nonself proteins, in recent years it has been shown that the oral or nasal administration of autoantigens is an effective means of suppressing autoimmune diseases in animals (4). Different mechanisms of tolerance are induced when proteins are given orally at different doses. Generally, as shown in Figure 1, higher doses induce anergy or deletion $(5,6)$, whereas lower doses induce active cellular regulation by stimulating $\mathrm{T}$ cells that secrete TGF- $\beta$, IL-10, or IL-4 (7).

Two papers -1 in this issue and 1 in a current issue of the JCI - report the treatment of experimental autoimmune myasthenia gravis (EAMG) by induction of oral tolerance to AChR in mice and rats $(8,9)$. The successful treatment of EAMG by mucosally administered (oral and nasal) AChR has been described previously (10-13). The 2 new papers contribute significantly to our ultimate ability to apply oral tolerance to the human disease. Im et al. (8) describe the suppression of EAMG by oral administration of the AChR recombinant fragment Ho1-205. Most important, they report that the recombinant fragment can successfully treat EAMG not only when it is given before disease induction or during the acute phase of the disease, but also when it is given during the chronic phase, when the ani- mal disease very closely mimics the human illness. The mechanism of disease suppression is the induction of active cellular regulation because oral administration of the Ho1-205 fragment induces cells that can adoptively transfer disease suppression to other animals. Furthermore, oral treatment is associated with immune responses characterized by a decrease of Th1-type responses (e.g., production of IL-12 and IFN- $\gamma$ ), and an increase of Th2-type and Th3-type responses (production of IL-10 and TGF- $\beta$, respectively). Oral antigen preferentially induces TGF- $\beta$-type responses, because TGF- $\beta$ serves as a switch factor for IgA in the gut (14). Th1-type anti-AChR antibody levels are also decreased. The H $\alpha 1-205$ fragment was administered without a fusion partner, thus making it applicable to treating humans. Furthermore, oral administration did not lead to priming for autoantibodies. Baggi et al. describe the suppression of EAMG by oral administration of To146-162, a 17-amino-acid T-cell epitope of the $\alpha$ chain of the AChR (9). Its disease-modulating effect is seen with relatively high doses, and its mechanism of action appears to be the induction of anergy or deletion, because no evidence of active cellular regulation or immune deviation was found in orally

\section{Figure 1}

The different mechanisms of oral tolerance are determined by the dose of fed antigen. GALT, gut-associated lymphoid tissue; TGF- $\beta$, transforming growth factor- $\beta$; Th, T helper.

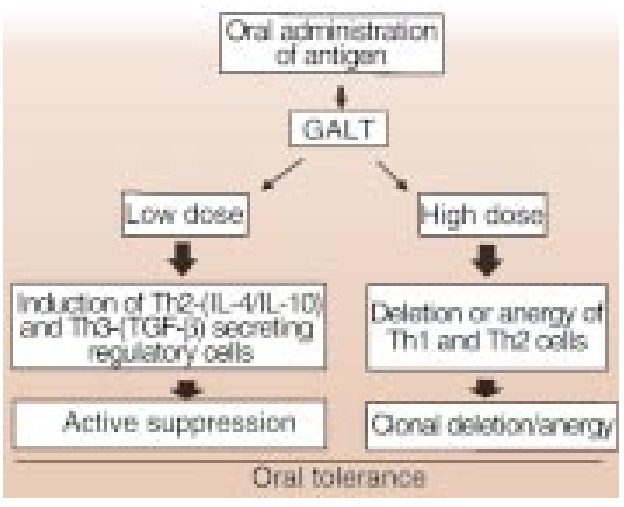


treated animals. Nonetheless, as with oral treatment with $\mathrm{H} \alpha 1-205$, there was no exacerbation of disease, and a decrease in Th1-type AChR antibodies was observed. Thus, Baggi, et al. demonstrate the suppression of EAMG by a different mechanism when a T-cell epitope is given by the oral route.

The immediate question raised by these studies is whether inducing oral tolerance with either a nonmyasthenogenic fragment of the $\alpha$ chain of the AChR or a specific T-cell epitope of the AChR could be successfully applied to the human disease. These studies demonstrate that fragments of the AChR or a T-cell epitope may be used to suppress the entire disease process, obviating the need for administering the entire AChR (which might have the undesirable effect of enhancing antibody responses). They also demonstrate the T-cell dependency of antibody responses, and that antibody-mediated autoimmune diseases such as myasthenia gravis may be treated by oral administration of a protein that affects the action of $\mathrm{T}$ cells in providing help for antibody responses.

Oral administration of autoantigens is being tested in human autoimmune diseases, but has not yet led to successful clinical application (4). Currently, major trials are being conducted in juvenile diabetes in which oral insulin is being administered, and a large phase III trial of oral glatiramer acetate (a myelin basic protein analogue) is being undertaken in multiple sclerosis (15). One difficulty in applying oral tolerance to human diseases has been the lack of an immune marker that could be used to monitor therapy and to demonstrate not only that oral tolerance has indeed been induced, but that the pathogenic immune response has been affected. In this regard, myasthenia gravis -1 of the best-characterized human autoimmune diseases - is especially well suited to investigations of antigen-specific immunotherapeutic approaches such as oral tolerance, because both anti-AChR antibodies and clinical responses are easily measured. Furthermore, it may or may not make a difference clinically whether anergy, deletion, or active cellular regulation are induced; both mechanisms have been shown to be effective in suppressing anti-AChR antibodies and ameliorating clinical disease in animals. Although worsening of autoimmune diseases after mucosal antigen treatment has been reported in some experimental systems (16-18), such worsening is the exception rather than the rule, and the large-scale human trials to date have not found worsening of disease when autoantigens were given orally. Therefore, the results presented by Im et al. (8) and Baggi et al. (9) provide the basis for the application of induced oral tolerance to the AChR for the antigenspecific treatment of myasthenia gravis.

1. Drachman, D.B. 1994. Myasthenia gravis. N. Engl. J. Med. 330:1797-1810.

2. Toyka, K.V., et al. 1977. Myasthenia gravis. Study of humoral immune mechanisms by passive transfer to mice. N. Engl. J. Med. 296:125-131.

3. Mowat, A.M. 1987. The regulation of immune responses to dietary protein antigens. Immunol. Today. 8:93-98.

4. Faria, A.M.C., and Weiner, H.L. 1999. Oral tolerance: mechanisms and therapeutic applications. Adv. Immunol. 73:153-264.

5. Whitacre, C.C., Gienapp, I.E., Orosz, C.G., and Bitar, D. 1991. Oral tolerance in experimental autoimmune encephalomyelitis. III. Evidence for clonal anergy. J. Immunol. 147:2155-2163.

6. Chen, Y., et al. 1995. Peripheral deletion of antigen-reactive $\mathrm{T}$ cells in oral tolerance. Nature. 376:177-180.

7. Chen, Y., Kuchroo, V.K., Inobe, J.-I., Hafler, D.A., and Weiner, H.L. 1994. Regulatory T-cell clones induced by oral tolerance: suppression of autoimmune encephalomyelitis. Science. 265:1237-1240.

8. Im, S.-H., Barchan, D., Fuchs, S., and Souroujon, M.C. 1999. Suppression of ongoing experimental myasthenia by oral treatment with an acetylcholine receptor recombinant fragment. J. Clin. Invest. 104:1723-1730.

9. Baggi, F., et al. 1999. Oral administration of an immunodominant T-cell epitope downregulates Th1/Th2 cytokines and prevents experimental myasthenia gravis. J. Clin. Invest. 104:1287-1295.

10. Wang, Z.Y., Qiao, J., and Link, H. 1993. Suppression of experimental autoimmune myasthenia gravis by oral administration of acetylcholine receptor. J. Neuroimmunol. 44:209-214.

11. Okumura, S., McIntosh, K., and Drachman, D.B. 1994. Oral administration of acetylcholine receptor: effects on experimental myasthenia gravis. Ann. Neurol. 36:704-713.

12. Ma, C.-G., et al. 1995. Suppression of experimental autoimmune myasthenia gravis by nasal administration of acetylcholine receptor. J. Neuroimmunol. 58:51-60.

13. Karachunski, P.I., Ostlie, N.S., Okita, D.K., and Conti-Fine, B.M. 1997. Prevention of experimental myasthenia gravis by nasal administration of synthetic acetylcholine receptor $\mathrm{T}$ epitope sequences. J. Clin. Invest. 100:3027-3035.

14. Kim, P.-H., and Kagnoff, M.F. 1990. Transforming growth factor- $\beta 1$ is a costimulator for $\operatorname{IgA}$ production. J. Immunol. 144:3411-3416.

15. Weiner, H.L. 1999. Oral tolerance with copolymer 1 for the treatment of multiple sclerosis. Proc. Natl. Acad. Sci. USA. 96:3333-3335.

16. Miller, A., Lider, O., Abramsky, O., and Weiner, H.L. 1994. Orally administered myelin basic protein in neonates primes for immune responses and enhances experimental autoimmune encephalomyelitis in adult animals. Eur. J. Immunol. 24:1026-1032.

17. Blanas, E., Carbone, F.R., Allison, J., Miller, J.F.A.P., and Heath, W.R. 1996. Induction of autoimmune diabetes by oral administration of autoantigen. Science. 274:1707-1709.

18. Hu, W., et al. 1998. Experimental mucosal induction of uveitis with the $60-\mathrm{kDa}$ heat shock protein-derived peptide 336-351. Eur. J. Immunol. 28:2444-2455. 